Research Article

\title{
A Multichannel Allocation Strategy Based on Preemption Threshold and Preemption Probability in Cognitive Radio Networks
}

\author{
Yuan Zhao $(i)$ and Zhiyu Xiang \\ School of Computer and Communication Engineering, Northeastern University at Qinhuangdao, Qinhuangdao, China \\ Correspondence should be addressed to Yuan Zhao; yuanzh85@163.com
}

Received 18 June 2021; Revised 19 August 2021; Accepted 7 September 2021; Published 24 September 2021

Academic Editor: Adrian Kliks

Copyright ( $\odot 2021$ Yuan Zhao and Zhiyu Xiang. This is an open access article distributed under the Creative Commons Attribution License, which permits unrestricted use, distribution, and reproduction in any medium, provided the original work is properly cited.

\begin{abstract}
In traditional multichannel cognitive radio networks (CRNs), users are split into two different priorities. Because of the low priority of secondary users (SUs), SU packets' transmissions are easily interrupted by primary users (PUs). In this paper, two control parameters, called preemption threshold $H$ and preemption probability $q$, are used to regulate the preemption behavior of PU packets to improve the transmission performance of SU packets. When all channels in the system are occupied, the preemption behavior of PU packets will be adjusted according to the amount of SU packets that are transmitting in the system. If the amount is larger than $H$, the recently arrived PU packet either preempts a channel with probability $q$ or leaves the system with probability $(1-q)$. The central controller manages the system's channel usage right and determines a series of access behaviors of user packets. Considering the possible imperfect sensing, a discrete-time queueing model is developed with the proposed preemption control mechanism. Then we obtain some performance index expressions of PU and SU packets founded on the system's state transition matrix and make the corresponding performance figures through numerical experiment. Finally, we construct a system utility function and determine the optimal preemption threshold and preemption probability through the seagull optimization algorithm (SOA). Experimental data show that the proposed mechanism by setting preemption threshold and preemption probability can significantly reduce SU packets' outage rate and improve SU packets' throughput rate.
\end{abstract}

\section{Introduction}

The scarcity of spectral resources has become more apparent in recent years, as wireless networking technology has advanced and the need for data transmission has grown. Researchers have found that the depletion of spectral resources is just an illusion, while the root cause is low spectral utilization [1-3]. As one of the critical technologies that can make rational use of spectral resources and effectively improve spectral utilization, cognitive radio (CR) has become a research hotspot [4]. It can sense the surrounding environment, reconfigure the relevant parameters according to the network load, and use dynamic spectrum access (DSA) to enable unauthorized SUs to use the idle spectrum opportunistically without interference to authorized PUs so as to solve the problem of insufficient spectrum resources $[5,6]$.
The most important access mode of DSA is called overlay mode, and each user packet can occupy a single channel to transmit data at the highest rate in this access mode $[7,8]$. But, with the lower priority, SU packets can only opportunistically occupy the idle channels that are not used by PUs and need to sense whether there are new PU packets in real time during the transmission process. Once a new PU packet is found to arrive, the current transmission of this SU packet should be stopped immediately, and the channel usage right should be given to the recently arrived PU packet [9]. When interrupt events in the system occur frequently, this will not only increase the comprehensive SUs' outage rate in the system and bring bad user transmission experience but also greatly reduce the throughput of the two types of users and eventually lead to a sharp decline in the overall performance of the system. 
Therefore, choosing a reasonable access control technology to regulate the two types of users' access behaviors is necessary. Recently, coordinated dynamic spectrum management has attracted the interest of some researchers $[10,11]$. In this method, the two types of users are not completely transparent. The access behavior of PUs will be adjusted according to the access activities of SUs. As long as there are still unoccupied channels in the system, the transmission of SUs will not be interrupted. Therefore, this type of access control scheme is of great value in improving SUs' performance in cognitive radio networks.

To increase the transmission efficiency of SUs, some scholars begin to focus on channel reservation. In [12], a coordinated channel reservation model for SUs was studied by using a Markov model. The system performance with and without reserved channels was compared, and the effect of the number of reserved channels on system efficiency was analyzed. In [13], considering the diversity of SUs, a dynamic channel reservation and channel aggregation scheme for hierarchical SUs was implemented to reduce SUs' starvation rate and frequency of channel switching. This scheme increased bandwidth usage effectively. In [14], a new dynamic channel reservation algorithm and spectrum access algorithm were introduced to study the network unavailability probability, bandwidth, service retention rate, and channel availability. The findings showed that the proposed algorithms could reduce the switching and blocking possibility by using the available spectrum effectively.

Controlling the preemption behavior is another way to ensure the transmission continuity of SUs. In [15], considering hierarchical SUs, a service principle combining preemption and nonpreemption was proposed. When the number of interruptions of low-level SUs reached the threshold, the high-level SUs would no longer preempt the low-level SUs, while the PUs could always preempt any kind of SUs. Experimental results showed that low-level SUs could get better performance by adjusting the threshold. In [16], a single-channel access strategy based on preemption probability was proposed. The preemption behavior of PU packets was controlled by changing the value of preemption probability, and the amount of interrupted SU packets was reduced by setting an appropriate preemption probability.

The concept of channel reservation, according to the above literature, can greatly increase SU packets' transmission performance. However, this idea regulates that a certain type of users can only use some special channels and cannot improve the spectrum utilization well. In other words, the essence of channel reservation is to reduce the outage rate by increasing the blocking rate. Different from mentioned literature, we consider enhancing SU packets' transmission performance by regulating PU packets' preemption behavior. All channels in the system can be shared by the two groups of users in a centralized network, and a preemption case arises only when the amount of SU packets reaches a certain level. This ensures the quality of service (QoS) of the authorized PU packets and effectively improves the transmission performance of the unauthorized SU packets. Different from literature [16], we introduce a preemption threshold based on the preemption probability to limit the preemption conditions of PU packets. In other words, the preemption threshold determines whether the PU packets can initiate preemption, and the preemption probability determines whether the PU packets can preempt successfully. Besides, to better adapt to the network's complexity, we consider and analyze a network system with multiple channels rather than a single channel. Therefore, the mechanism proposed in this paper not only retains the advantages of preemption probability proposed in literature [16] but also introduces a preemption threshold to improve the sensitivity and adjustability of the model to meet the needs of more complex networks.

The main contributions of our paper are summarized as follows:

(1) A centralized DSA technique that incorporates two new parameters, preemption threshold and preemption probability, which are used to manage the preemption activity of PU packets dynamically, is proposed.

(2) A discrete-time queueing model is constructed with the proposed preemption control scheme, and some performance index expressions are derived.

(3) Numerical experiments are conducted to evaluate the performance of the proposed preemption control mechanism. The effectiveness of the preemption control mechanism on improving the system performance is shown with numerical results.

(4) An optimization scheme is given to find out the optimal preemption threshold and the optimal preemption probability by considering the trade-off of the performance of PU and SU.

The rest of our paper is set out as follows. We propose a new type of preemption control strategy based on imperfect spectrum state sensing and establish a discrete-time queueing model in Section 2. Some system performance index expressions are shown in Section 3. In Section 4, numerical results show the impact of preemption threshold and preemption probability on system performance. We give a proper system utility function by weighing two important performance indicators and get the optimal parameters through optimization experiments in Section 5. Finally, in Section 6, we summarize the work done in this paper.

\section{System Model}

2.1. Mechanism Description. Figure 1 shows the architecture of a centralized cognitive radio network [12] considered in this paper.

As Figure 1 shows, there is one $\mathrm{PU}$, one $\mathrm{SU}$, and $M$ data transmission channels in the system, and a central controller manages these channels. Each data transmission channel has the same transmission capability for the same type of user packets and can serve one user packet at a time. Each user can generate multiple packets, but each type of user can generate at most one new packet in a time slot. PU packets can preempt the channel usage right of unauthorized SU packets because of their high priority. Once the SU packet 


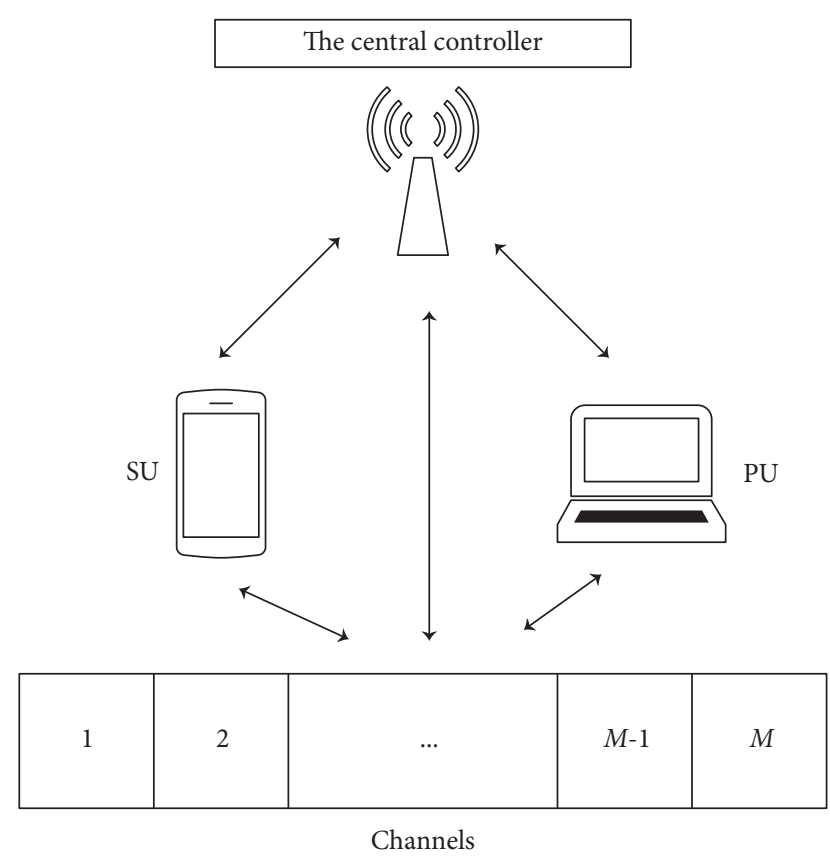

FIGURE 1: Cognitive radio network with a centralized architecture.

transmitting on the channel detects a PU packet that initiates preemption, it must immediately give up the channel usage right. Two parameters are introduced to control the preemption behavior of PU packets, namely, preemption threshold $H(H<M)$ and preemption probability $q(0<q \leq 1)$. The central controller maintains a database to record the values of state variables in the system and the channel occupation information of PU and SU packets. At the beginning of each time slot, the central controller will update the database according to the real-time state of the system and allocate idle channels for the packets arriving at the moment. The central controller firstly processes the arrival request of $\mathrm{PU}$ packets to ensure the priority of the PU. In the mechanism proposed in this paper, when all of the channels are occupied, a recently arrived SU packet will be directly rejected by the central controller to access the system, and, for a recently arrived PU packet, whether or not to occupy a channel used by an SU packet according to the value of the preemption threshold and the preemption probability will be determined by the central controller.

Figure 2 describes the access behavior and transmission behavior of two types of users to show the mechanism set out in the present paper more clearly.

When a recently arrived PU packet needs to enter the system for data transmission, if there is more than one unoccupied channel, it will occupy one randomly for data transmission; if there are no idle channels, the central controller will decide whether to allow this PU packet to preempt a channel according to the amount of SU packets that are transmitting in the system. However, if the amount is less than or equal to the preemption threshold $H$, the recently arrived PU packet cannot preempt any channel using by SU packets; if the amount exceeds threshold $H$, the recently arrived PU packet either preempts with probability $q$ or leaves with probability $(1-q)$.
When a recently arrived SU packet needs to enter the system for data transmission, if there is more than one idle channel in the system at this moment, it will occupy one randomly for transmission; if there is only one idle channel, it will only use this channel when no PU packet accesses at this time, or it will be blocked and discarded by the system; if all the channels are busy at this time, it will leave the system immediately. Besides, each SU packet transmitting in the system needs to listen to the external situation. Once there is a PU packet that initiates preemption against itself, it needs to stop the transmission and deliver the channel usage right to this PU packet.

Considering the fact that the amount of SU packets in the system changes frequently, the central controller in practice may misperceive the actual amount of SU packets and make wrong preemption decisions. We assume that there are two types of false perceptions that may occur in the mechanism we propose. While the actual amount of SU packets is less than or equal to threshold $H$, the perceived amount of SU packets may exceed $H$; then the recently arrived PU packet may preempt the channel by mistake. We use $f_{1}\left(\bar{f}_{1}=1-\right.$ $\left.f_{1}, 0<f_{1}<1\right)$ to express the probability of this condition. On the contrary, we use $f_{2}\left(\bar{f}_{2}=1-f_{2}, 0<f_{2}<1\right)$ to represent the probability that the actual amount exceeds the threshold and the observed value is less than or equal to the threshold value by mistake. By introducing these two probability parameters, we can better adapt to the actual situation.

2.2. Model Building. We abstract the $M$ data transmission channels in the system to $M$ service stations, which have the same transmission ability, and divide the time axis into discrete slots with the same length. It is assumed that PU and SU packets are customers in the queueing system and the arrival intervals follow geometric distribution, and arrival rates are $p_{1}\left(\bar{p}_{1}=1-p_{1}, 0<p_{1}<1\right), p_{2}\left(\bar{p}_{2}=1\right.$ $\left.-p_{2}, 0<p_{2}<1\right)$, respectively. The service time of two groups of packets obeys geometric distribution as well, and service rates are $\mu_{1}\left(\bar{\mu}_{1}=1-\mu_{1}, 0<\mu_{1}<1\right), \mu_{2}\left(\bar{\mu}_{2}=1-\right.$ $\left.\mu_{2}, 0<\mu_{2}<1\right)$, respectively. According to the Early Arrival System (EAS) assumption, each service completion of user packets occurs at the end of the slot $\left(t^{-}, t\right)$, and each arrival occurs at the beginning of the slot $\left(t, t^{+}\right)$[17]. We mark PU packets' amount at time $t^{+}$as $P_{t}$ and SU packets' amount at time $t^{+}$as $S_{t}$ and call $P_{t}$ system level and $S_{t}$ system phase. So $\left\{P_{t}, S_{t}: t \geq 0\right\}$ constitutes a 2 -dimensional Markov chain (2DMC) with state-space $\mathbf{T}$; $\mathbf{T}$ is defined as follows:

$$
\mathbf{T}=\{(m, n): 0 \leq m \leq M, 0 \leq n \leq M\}
$$

In the above formula, $m$ and $n$ are the two state variables of the system. We use $\mathbf{E}$ to represent the system's one-step transition matrix, use $(i, j)\{(i, j) \in \mathbf{T}\}$ to represent the state before the state transition, and use $(k, l)\{(k, l) \in \mathbf{T}\}$ to represent the state after the state transition. Therefore, the probability of one-step transition from state $(i, j)$ to $(k, l)$ is denoted by $E_{(i, j) \rightarrow(k, l)}$, which is expressed as 


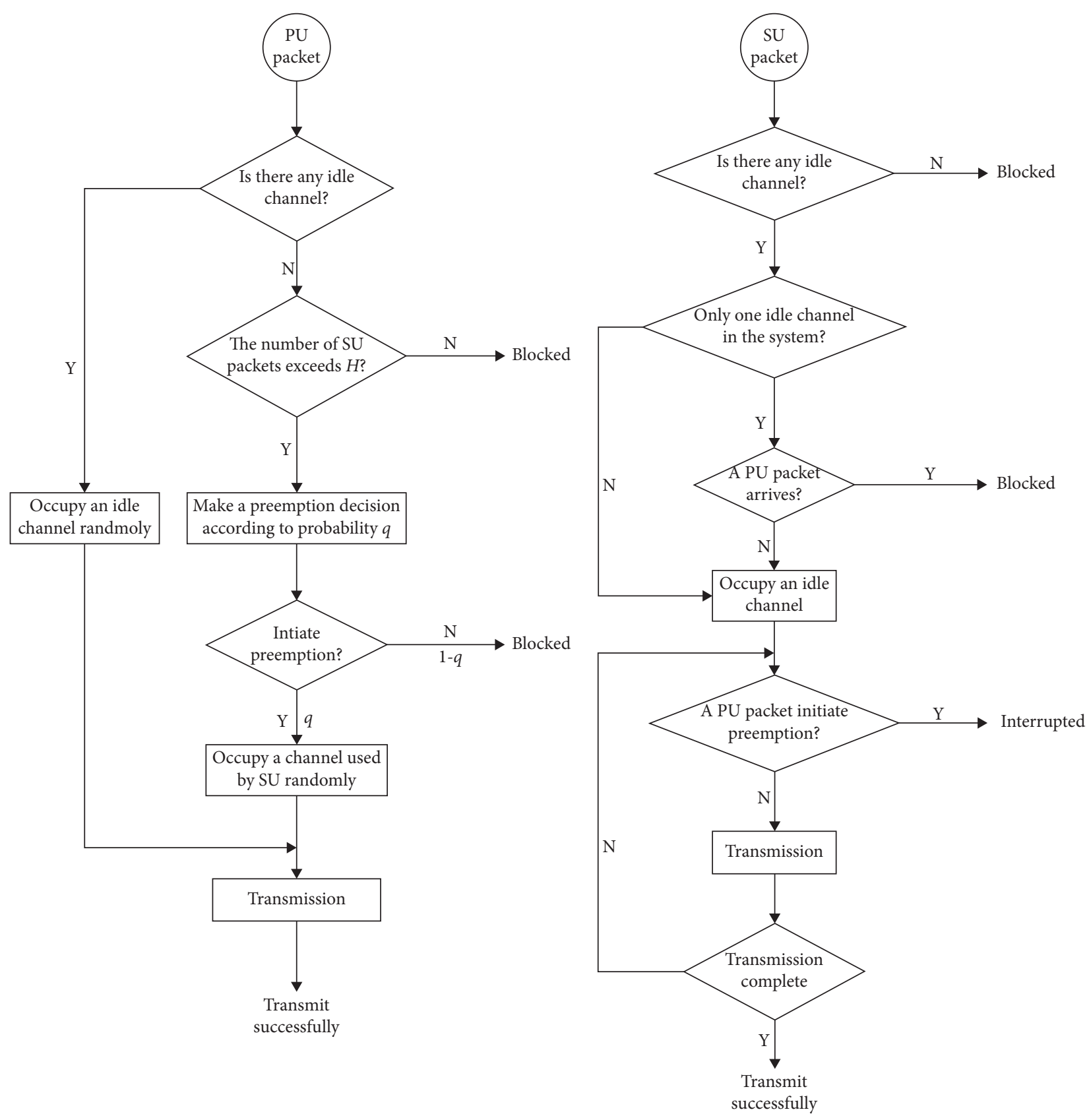

FIgURE 2: The system behavior of two types of users.

$$
E_{(i, j) \longrightarrow(k, l)}= \begin{cases}0, & (i+j)>M \text { or }(k+l)>M \text { or }(k-i)>1 \text { or }(l-j)>1, \\ A_{i, k} B_{j, l}+R, & \text { else. }\end{cases}
$$

In the above formula, we divide the transition probability into two parts according to whether preemption occurs. $A_{i, k} B_{j, l}$ represents the probability when there is no preemption; $A_{i, k}$ is the probability from level $i$ to $k$, and $B_{j, l}$ is the probability from phase $j$ to $l$. When the state after transition $(k, l)$ satisfies $(k+l)=M$, it means that, after the one-step transition, there are no idle channels in the system, and preemptive events may occur at this time. Therefore, we add $R$ to represent the preemption probability or the probability due to unsuccessful preemption. 
To express the transition matrix $\mathbf{E}$ concisely, we define two probability variables called $\beta_{1}$ and $\beta_{2}$, which will be used in this section.

$$
\begin{aligned}
& \beta_{1}=\left(\begin{array}{c}
i \\
i-k
\end{array}\right) \bar{p}_{1} \mu_{1}^{i-k} \bar{\mu}_{1}^{k}+\left(\begin{array}{c}
i \\
i-k+1
\end{array}\right) p_{1} \mu_{1}^{i-k+1} \bar{\mu}_{1}^{k-1}, \\
& \beta_{2}=\left(\begin{array}{c}
j \\
j-l
\end{array}\right) \bar{p}_{2} \mu_{2}^{j-l} \bar{\mu}_{2}^{l}+\left(\begin{array}{c}
j \\
j-l+1
\end{array}\right) p_{2} \mu_{2}^{j-l+1} \bar{\mu}_{2}^{l-1},
\end{aligned}
$$

where the formula represented as $\left(\begin{array}{l}b \\ a\end{array}\right)$ is defined as follows:

$$
\left(\begin{array}{l}
b \\
a
\end{array}\right)= \begin{cases}\frac{b !}{a !(b-a) !}, & 0 \leq a \leq b, \\
0, & \text { else. }\end{cases}
$$

We will discuss the transition matrix represented by equation (2) in detail.

(1) Since the network has $M$ channels, the maximum amount of PU and SU packets that can be accommodated is $M$, and each type of packets can only reach one in the same time slot. So when $(i+j)>M$ or $(k+l)>M$ or $(k-i)>1$ or $(l-j)>1$, $E_{(i, j) \longrightarrow(k, l)}=0$.

(2) When the above conditions are not met, if $k+l<M$, that is, after the one-step transition, there are still idle channels in the system. So, in this condition, there must be no preemption and blocking, and the behaviors of two groups of users are independent of each other. $A_{i, k}, B_{j, l}$, and $R$ are expressed as follows:

$$
\begin{gathered}
A_{i, k}= \begin{cases}p_{1} \mu_{1}^{i}, & k=i+1, \\
\beta_{1}, & k \leq i,\end{cases} \\
B_{j, l}= \begin{cases}p_{2} \bar{\mu}_{2}^{j}, & l=j+1, \\
\beta_{2}, & l \leq j,\end{cases} \\
R=0 .
\end{gathered}
$$

(3) Similarly, when the first condition is not met, if $k+l=M$, that is, after the one-step transition, there are no idle channels in the system. So, in this condition, preemption and blocking may occur in the system. We will discuss it according to the following four situations:

(1) When $k=i+1, l=j$, that is, after the one-step transition, the amount of PU packets increases by one, while the amount of SU packets remains unchanged. In this instance, the SU packets that are transmitting in the channels are not preempted by the recently arrived PU packet. So $A_{i, k}, B_{j, l}$, and $R$ are expressed as follows:

$$
\begin{aligned}
A_{i, k} & =p_{1} \bar{\mu}_{1}^{i}, \\
B_{j, l} & =\beta_{2}+p_{2} \bar{\mu}_{2}^{j},
\end{aligned}
$$

$$
R=0 \text {. }
$$

(2) When $k=i+1, l=j-1$, that is, after the onestep transition, the amount of PU packets increases by one, while the amount of SU packets decreases by one. In this case, $A_{i, k}$ and $B_{j, l}$ are expressed as follows:

$$
\begin{aligned}
& A_{i, k}=p_{1} \bar{\mu}_{1}^{i}, \\
& B_{j, l}=\beta_{2}+j p_{2} \mu_{2} \bar{\mu}_{2}^{j-1} .
\end{aligned}
$$

According to the amount of SU packets, the two following situations are discussed:

(a) When $j \leq H$, that is, before the one-step transition, the amount of SU packets does not exceed the threshold. So, in this case, considering the imperfect perception introduced in Subsect. 2.1, $R$ is expressed as follows:

$$
R=p_{1} \bar{\mu}_{1}^{i} \bar{\mu}_{2}^{j} f_{1} q .
$$

(b) When $j>H$, that is, before the one-step transition, the amount of SU packets exceeds the value of the threshold. So, in this case, $R$ is expressed as follows:

$$
R=p_{1} \bar{\mu}_{1}^{i} \bar{\mu}_{2}^{j} \bar{f}_{2} q .
$$

(3) When $k=i, l=j$, that is, after the one-step transition, the amount of two kinds of packets remains unchanged. On this condition, the SU packets which are transmitting in the channels are not preempted by the recently arrived PU packet. In this distance, $A_{i, k}$ and $B_{j, l}$ are expressed as follows:

$$
\begin{aligned}
& A_{i, k}=\beta_{1}, \\
& B_{j, l}=\beta_{2}+p_{2} \bar{\mu}_{2}^{j} .
\end{aligned}
$$

(a) When $1 \leq j \leq H$, we can imitate the above derivation and write the following expression:

$$
R=p_{1} \bar{\mu}_{1}^{i} \bar{\mu}_{2}^{j}\left[f_{1}(1-q)+\bar{f}_{1}\right] .
$$

(b) When $0=j \leq H$, there are no SU packets transmitting in the channels before the state transition. In this distance, even if the wrong perception occurs, preemption is impossible. $R$ is expressed as follows:

$$
R=p_{1} \bar{\mu}_{1}^{i} \bar{\mu}_{2}^{j} \text {. }
$$

(c) When $j>H, R$ is expressed as follows:

$$
R=p_{1} \bar{\mu}_{1}^{i} \bar{\mu}_{2}^{j}\left[f_{2}+\bar{f}_{2}(1-q)\right] \text {. }
$$

(4) When $l=j+1, A_{i, k}, B_{j, l}$, and $R$ are expressed as follows: 


$$
\begin{aligned}
A_{i, k} & = \begin{cases}p_{1} \bar{\mu}_{1}^{i}, & k=i+1, \\
\beta_{1}, & k \leq i,\end{cases} \\
B_{j, l} & =p_{2} \bar{\mu}_{2}^{j}, \\
R & =0 .
\end{aligned}
$$

So far, the state transition matrix $\mathbf{E}$ has been wholly expressed. By referring to literature [18], the steady-state probability distribution of the system is solvable. We define the steady-state distribution $\pi_{m, n}$ of the 2DMC $\left\{P_{t}, S_{t}: t \geq 0\right\}$ as follows:

$$
\pi_{m, n}=\lim _{t \longrightarrow \infty} P\left\{P_{t}=m, S_{t}=n: 0 \leq m \leq M, 0 \leq n \leq M\right\} .
$$

We define $\boldsymbol{\Pi}=\left(\pi_{0,0}, \pi_{0,1}, \pi_{0,2}, \ldots, \pi_{M, M-1}, \pi_{M, M}\right)$ as the steady-state probability vector. According to the system equilibrium conditions and normalization conditions, the following equations can be obtained [19]:

$$
\left\{\begin{array}{l}
\boldsymbol{\Pi E}=\boldsymbol{\Pi}, \\
\sum_{x=0}^{M} \sum_{y=0}^{M} \pi_{x, y}=1 .
\end{array}\right.
$$

In the above equations, $x$ and $y$ are two state variables. By solving the equations, we obtain the value of $\Pi$.

\section{Performance Metrics}

To express performance metrics concisely, we define some mathematical symbols which will be used later.

$$
\begin{aligned}
& Y= \begin{cases}1, & M-x=0, \\
(1-q) f_{1}+\bar{f}_{1}, & 1 \leq M-x \leq H, \\
(1-q) \bar{f}_{2}+f_{2}, & M-x>H,\end{cases} \\
& F= \begin{cases}f_{1}, & M-x \leq H, \\
\bar{f}_{2}, & M-x>H .\end{cases}
\end{aligned}
$$

In the above equations, $x$ is an index variable.

3.1. PU Packets' Performance Metrics. PU packets' average queue length refers to the average amount of PU packets in the system in unit time, which is described as follows:

$$
E[\mathrm{PU}]=\sum_{x=0}^{M} \sum_{y=0}^{M-x} x \pi_{x, y} .
$$

The amount of PU packets that initiate access requests but fail to enter the system in unit time is defined as PU packets' blocking rate, and the form is as follows:

$$
\beta_{p u}=\sum_{x=0}^{M} \pi_{x, M-x} \bar{\mu}_{1}^{x} \bar{\mu}_{2}^{M-x} p_{1} Y .
$$

The total amount of PU packets that have completed data transmission in unit time is defined as PU packets' throughput rate, and the form is as follows:

$$
\theta_{\mathrm{pu}}=p_{1}-\beta_{\mathrm{pu}} \text {. }
$$

3.2. SU Packets' Performance Metrics. Similar to the performance indicators of PU packets described above, we can obtain SU packets' average queue length by analogy, and the form is expressed as follows:

$$
E[\mathrm{SU}]=\sum_{x=0}^{M} \sum_{y=0}^{M-x} y \pi_{x, y} .
$$

The amount of SU packets that are transmitting in the system interrupted by recently arrived PU packets in unit time is defined as SU packets' outage rate, and the form is as follows:

$$
\gamma_{\mathrm{su}}=\sum_{x=0}^{M-1} \pi_{x, M-x} \bar{\mu}_{1}^{x} \bar{\mu}_{2}^{M-x} p_{1} F q .
$$

SU packets' blocking rate is expressed as follows:

$$
\begin{aligned}
\beta_{s u}= & \sum_{x=0}^{M} \pi_{x, M-x} p_{2}\left[\bar{\mu}_{1}^{x} \bar{\mu}_{2}^{M-x}+x \mu_{1} \bar{\mu}_{1}^{x-1} \bar{\mu}_{2}^{M-x} p_{1}+\bar{\mu}_{1}^{x}(M-x) \mu_{2} \bar{\mu}_{2}^{M-x-1} p_{1}\right] \\
& +\sum_{x=0}^{M-1} \pi_{x, M-x-1} \bar{\mu}_{1}^{x} \bar{\mu}_{2}^{M-x-1} p_{1} p_{2} .
\end{aligned}
$$

Through the above analysis, SU packets' throughput rate is as follows:

$$
\theta_{\mathrm{su}}=p_{2}-\gamma_{\mathrm{su}}-\beta_{\mathrm{su}}
$$

SU packets' average latency refers to SU packets' average sojourn time from the arriving instant to the transmission completion instant or interrupted instant. According to Little's formula [20], it can be expressed as follows:

$$
\delta_{\mathrm{su}}=\frac{E[\mathrm{SU}]}{p_{2}-\beta_{\mathrm{su}}} .
$$

\section{Numerical Results}

4.1. Performance Analysis. To describe the system properties more clearly, we select some representative performance indicators and carry out numerical experiments on MAT$\mathrm{LAB}$ to get the change trends of relevant performance 
indicators. In different network environments, parameter settings are often different. The following are certain constant parameters in our experiment: $\mathrm{SU}$ packets' arrival rate is $p_{2}=0.6, \mathrm{PU}$ and SU packets' service rates are $\mu_{1}=0.1$ and $\mu_{2}=0.15$, respectively, and the two kinds of perception error rates are $f_{1}=0.15$ and $f_{2}=0.1$. The other dynamic parameters are indicated in the figures.

4.1.1. PU Packets' Performance Analysis. The mechanism with preemption threshold and preemption probability proposed in this paper can regulate the preemption behavior of PU packets, which may have a certain impact on PU packets' average queue length, blocking rate, and throughput rate.

Figures 3-5 illustrate the change trends of PU packets' average queue length, blocking rate, and throughput rate with preemption probability.

In Figures 3-5, as the preemption probability increases, PU packets' average queue length and throughput rate increase, and PU packets' blocking rate decreases. This is because a large preemption probability will make it easier for PU packets to preempt the channels, and fewer PU packets will be blocked in unit time slot. This will also increase the amount of PU packets for data transmission in the system, and then more PU packets can accomplish their transmission per unit time.

We can also find from Figures 3-5 that, under the same other conditions, a larger preemption threshold means a smaller PU packets' average queue length and a smaller throughput rate and also means a larger PU packets' blocking rate. This is because the preemption threshold restricts the preemption possibility of PU packets. Increasing the preemption threshold will limit the amount of PU packets entering the system. As a result, PU packets' average queue length and throughput rate reduce, and PU packets' blocking rate increases.

It can also be seen from Figures 3-5 that raising PU packets' arrival rate may also increase PU packets' average queue length, blocking rate, and throughput rate. It is because a higher PU packets' arrival rate ensures that more PU packets enter the system and complete the transmission per unit time. At the same time, there will be more PU packets blocked because the channels are all occupied. Therefore, these three performance indicators will all increase with PU packets' arrival rate increasing.

4.1.2. SU Packets' Performance Analysis. The proposed mechanism will also have a certain impact on SU packets' outage rate, throughput rate, and average latency.

Figures 6-8 illustrate the change trends of SU packets' outage rate, throughput rate, and average latency with preemption probability.

As shown in Figure 6, SU packets' outage rate increases with preemption probability growing. The explanation for this may be that more PU packets initiate preemption per unit time as the preemption probability increases, causing more SU packets to be interrupted. Furthermore, when the preemption threshold is large, SU packets' outage rate is low.

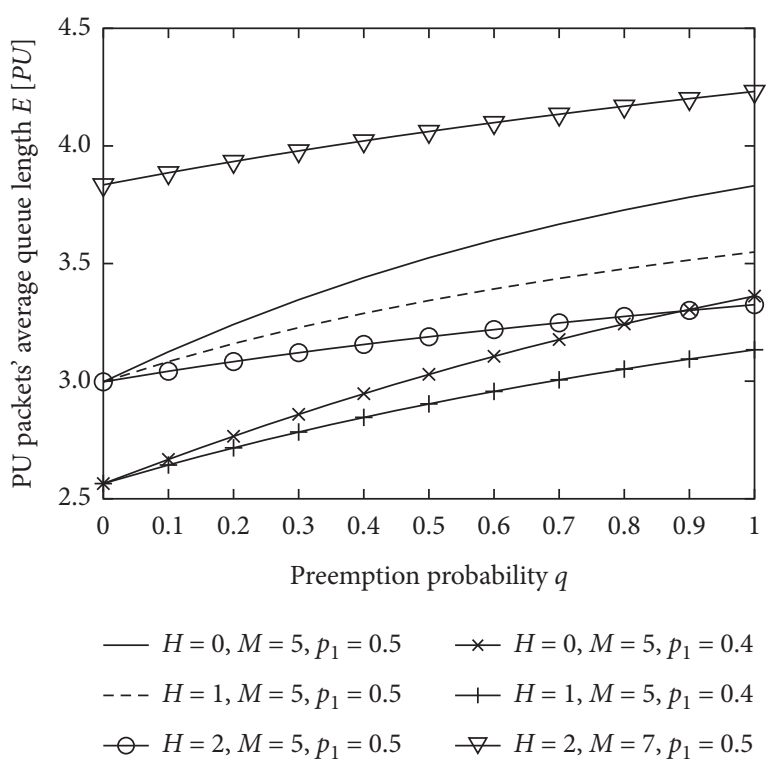

FIgURe 3: The trend in PU packets' average queue length.

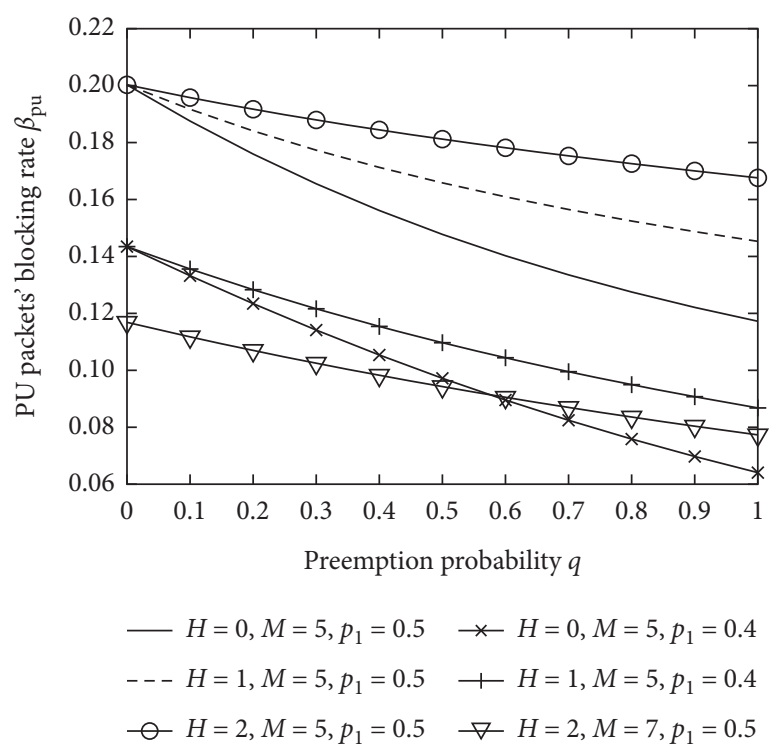

FIgURE 4: The trend in PU packets' blocking rate.

The explanation for this may be that the preemption threshold restricts $\mathrm{PU}$ packets' preemption behavior, and a larger threshold will allow fewer PU packets to initiate preemption. Besides, the higher the PU packets' arrival rate is, the more PU packets initiate preemption per unit time, so SU packets' outrage rate may be higher. We find that when the amount of channels is larger, SU packets' outage rate may be larger too. The explanation for this may be that the more channels, the more SU packets that can be accommodated in the system, resulting in more SU packets being interrupted.

As shown in Figure 7, with preemption probability increasing, SU packets' throughput rate decreases. It is because a large preemption probability will make it easier for PU packets to preempt. Accordingly, the amount of interrupted 


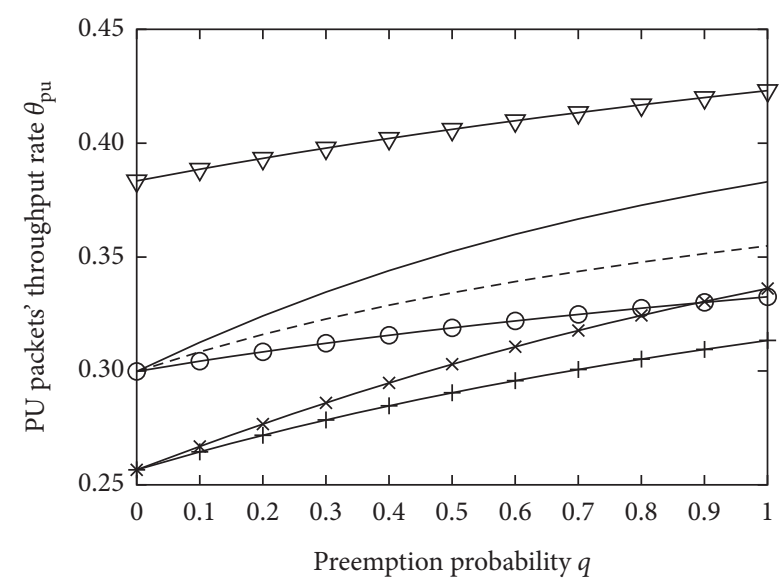

$$
\begin{array}{ll}
-H=0, M=5, p_{1}=0.5 & \rightarrow H=0, M=5, p_{1}=0.4 \\
--H=1, M=5, p_{1}=0.5 & \text { 十 } H=1, M=5, p_{1}=0.4 \\
-H=2, M=5, p_{1}=0.5 & \rightarrow H=2, M=7, p_{1}=0.5
\end{array}
$$

FIgURE 5: The trend in PU packets' throughput rate.

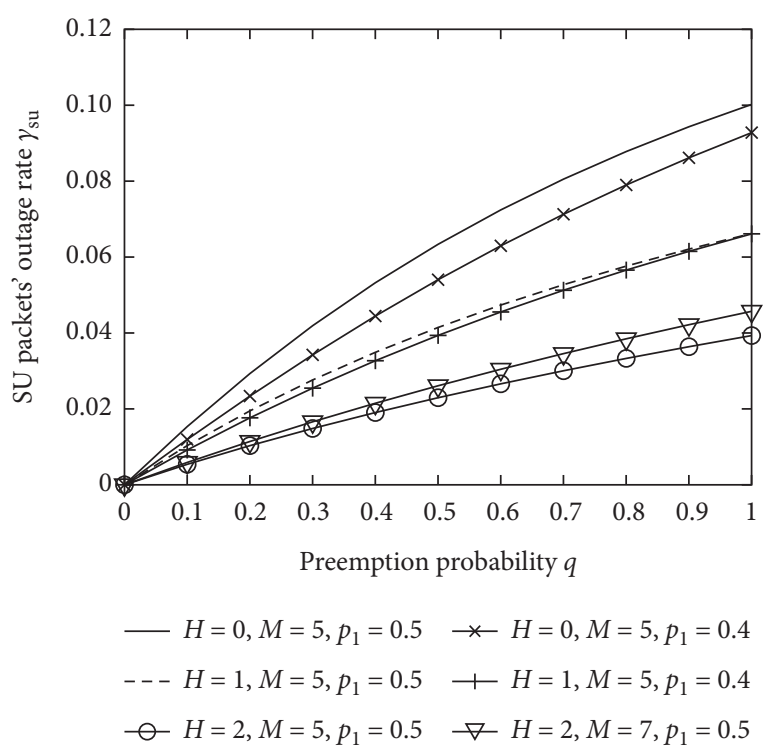

FIgURE 6: The trend in SU packets' outage rate.

SU packets will get larger, and SU packets' throughput rate will decrease. Besides, increasing the threshold will lead to an increased SU packets' throughput rate. It is because a larger threshold means a lower risk of SU packets being interrupted, so SU packets' throughput rate will increase with SU packets' outage rate decreasing. What is more, we note that the larger the PU packets' arrival rate is, the lower the SU packets' throughput rate will be. This is because the system's capacity is limited. When more PU packets arrive, the amount of channels used for SU packets will reduce. Moreover, if we set the amount of channels to be larger, SU packets' throughput rate will also be improved.

We can see from Figure 8 that SU packets' average latency decreases with the increase of preemption probability, since the larger the preemption probability is, the more interrupted

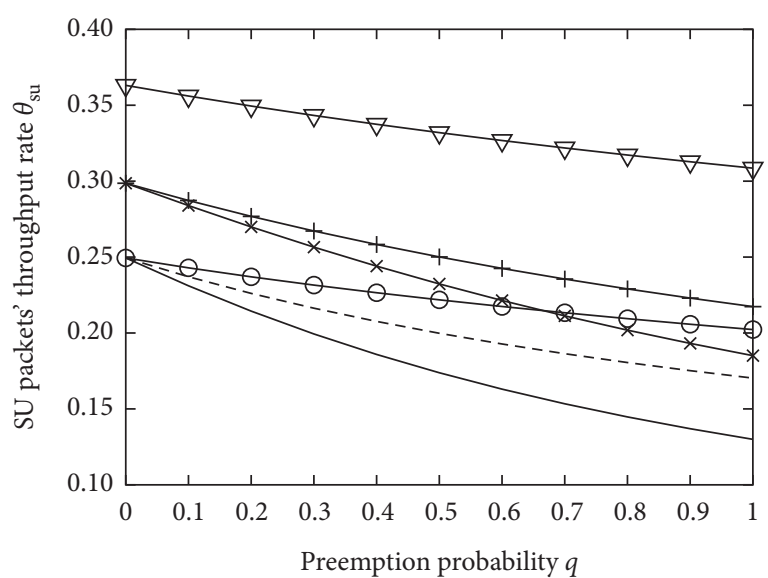

$$
\begin{array}{ll}
-H=0, M=5, p_{1}=0.5 & * H=0, M=5, p_{1}=0.4 \\
--H=1, M=5, p_{1}=0.5 & \rightarrow H=1, M=5, p_{1}=0.4 \\
-H=2, M=5, p_{1}=0.5 & \rightarrow H=2, M=7, p_{1}=0.5
\end{array}
$$

Figure 7: The trend in SU packets' throughput rate.

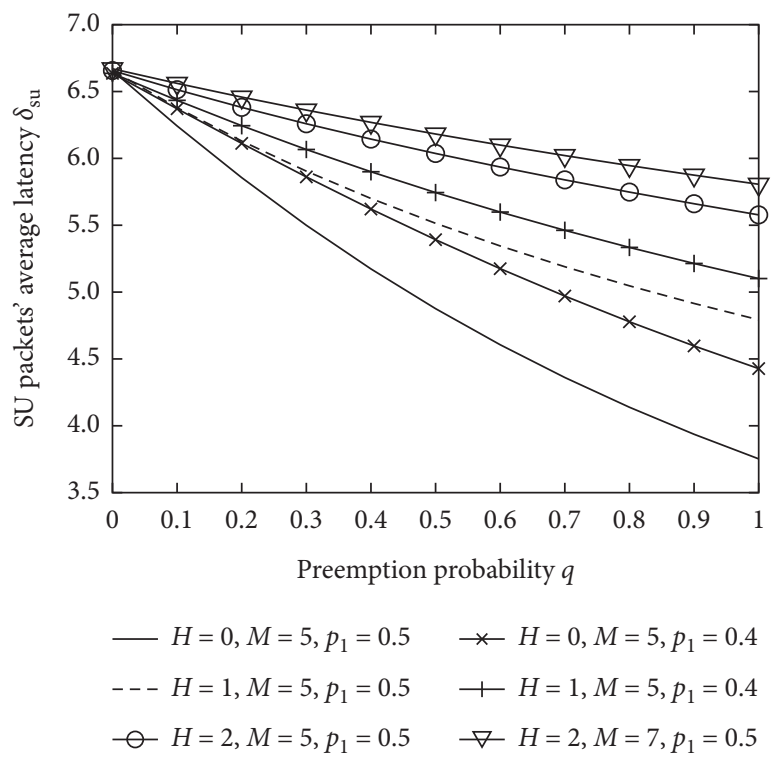

FIgURE 8: The trend in SU packets' average latency.

SU packets there will be, which leads to a shorter average transmission time. Besides, a larger preemption threshold corresponds to a larger average latency. It is just because the transmission quality of SU packets will be improved if the preemption threshold is set larger, the amount of SU packets that complete transmission will be more, and the amount of SU packets that leave the system early due to interruption will reduce, so the average transmission time in the system will be larger. Similarly, the increase in PU packets' arrival rate will also decrease the average latency. This is also due to a rise in the amount of interrupted SU packets. Besides, when the amount of channels is large, SU packets' average latency will also get large. When the amount of channels is large, more SU packets will successfully transmit per unit time in the system, resulting in a longer average time spent in the system. 
4.2. Performance Comparison. To more intuitively reflect the supremacy of the proposed mechanism, we will compare the change trends of the proposed mechanism with the traditional mechanism (the preemption threshold is 0 and the preemption probability is 1) and the mechanism proposed in literature [16] (the preemption threshold is 0 and the preemption probability is 0.8 ) from the perspective of PU and SU packets. The following are some of the constant parameters in this subsection: SU packets' arrival rate is $p_{2}=0.6, \mathrm{PU}$ and SU packets' service rates are $\mu_{1}=0.1$ and $\mu_{2}=0.15$, the amount of channels is $M=5$, and the two kinds of perception error rates are $f_{1}=0.15$ and $f_{2}=0.1$. The other dynamic parameters are shown in the Figures 9-11.

Figures 9-11 show the change trends of PU packets' blocking rate, SU packets' outage rate, and two kinds of packets' throughput rate with PU packets' arrival rate.

It can be seen from Figures 9-11 that the preemption control mechanism proposed in this paper can greatly improve the performance of SU packets at the expense of a slight decline for QoS of PU packets. Compared with the traditional mechanism (the preemption threshold is 0 and the preemption probability is 1), the performance of $\mathrm{PU}$ packets in our mechanism is not so good. However, the SU packets' outage rate is very low and the SU packets' throughput rate is significantly improved in our proposed mechanism. In addition, compared with the mechanism in literature [16] (the preemption threshold is 0 and the preemption probability is 0.8 ), the preemption threshold proposed in this paper can further improve the transmission continuity of SU packets and improve the sensitivity and adjustability of the model to adapt to more complex cognitive radio networks. Therefore, the mechanism we proposed can better meet the service needs of SU packets.

From Figure 10, we can see an interesting phenomenon that as PU packets' arrival rate grows, SU packets' outage rate first rises and then falls. This is because when PU packets' arrival rate is at a low level, the amount of SU packets transmitting in the channels is large. In this distance, the larger the PU packets' arrival rate is, the more preemptions will occur, and the larger the SU packets' outage rate will be. When PU packets' arrival rate is high, the amount of SU packets that can access the network is small. At this time, the higher the PU packets arrival rate is, the amount of SU packets transmitting in the channels will reduce, and the amount of SU packets that can be interrupted will also reduce.

Figure 11 shows the change trends of the two types of packets' throughput rates. With PU packets' arrival rate or preemption probability increasing, PU packets' throughput rate will increase, and SU packets' throughput rate will decrease. In addition, with the increase of the preemption threshold, the opposite is true. Therefore, we can conclude that there exists a trade-off between the throughput rates of the PU packets and SU packets.

\section{System Optimization}

5.1. Optimization Analysis. In practice, throughput rate is a representative index reflecting system transmission quality. From numerical results, we can see that PU packets'

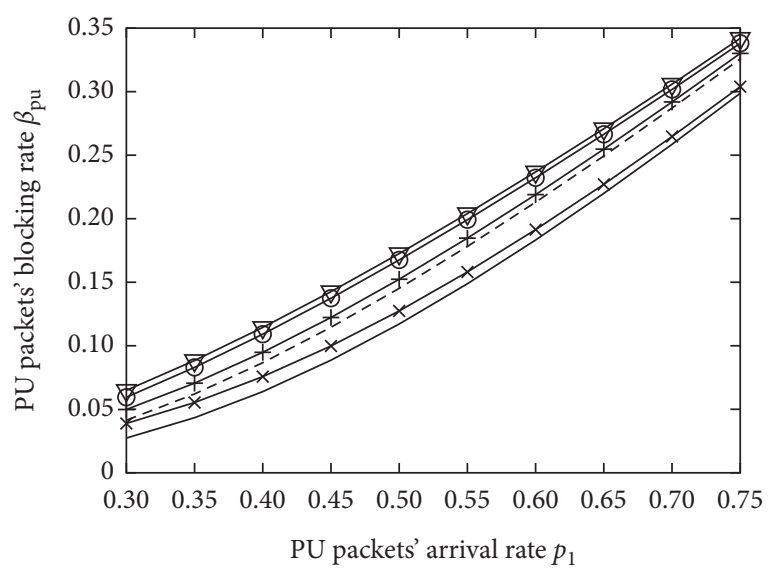

$$
\begin{array}{ll}
-H=0, q=1 & * H=0, q=0.8 \\
--H=1, q=1 & \rightarrow H=1, q=0.8 \\
-H=2, q=1 & \rightarrow H=2, q=0.8
\end{array}
$$

FIgURE 9: The trend in PU packets' blocking rate.

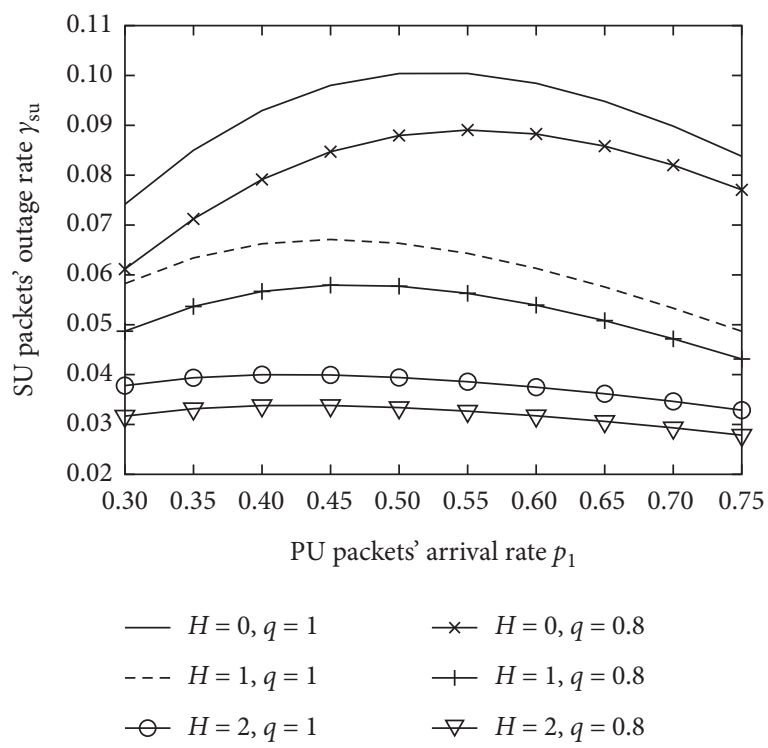

FIGURE 10: The trend in SU packets' outage rate.

throughput rate increases with the growth of preemption probability, which is what we desire to achieve. However, a larger preemption probability corresponds to a smaller SU packets' throughput rate. Similarly, for the preemption threshold, the larger the preemption threshold is, the larger the throughput of SU packets will be, but the lower the throughput of PU packets will be. Therefore, considering the throughput rate trade-off between PU and SU packets, to balance the two important performance indicators with opposite trends, we establish a utility function $U(H, q)$ to determine the optimal preemption threshold $H$ and preemption probability $q$. The utility function is defined as follows:

$$
U(H, q)=\alpha_{1} \theta_{\mathrm{pu}}+\alpha_{2} \theta_{\mathrm{su}} \text {. }
$$

In equation (36), $\alpha_{1}$ represents the system utility rewarded by unit PU packets' throughput rate, and $\alpha_{2}$ 


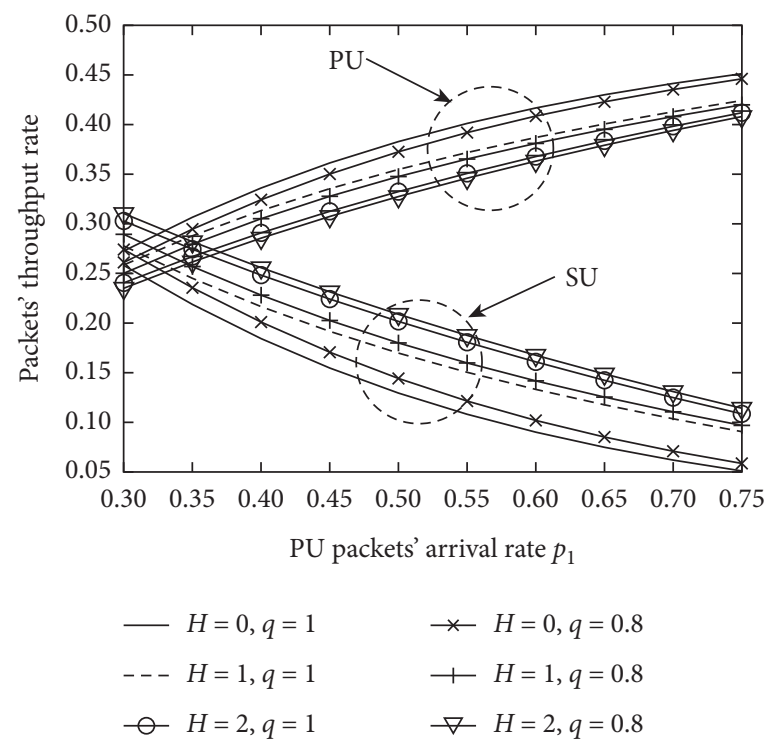

Figure 11: The trend in two kinds of packets' throughput rate.

represents the system utility rewarded by unit SU packets' throughput rate. We can adjust the settings for $\alpha_{1}$ and $\alpha_{2}$ according to different network operating environments. For example, in some leasing-based cognitive radio networks, PUs obtain certain benefits by renting spectrum resources to SUs. When the rent attached to SUs is high, we can increase the value of $\alpha_{2}$ or reduce the value of $\alpha_{1}$ to improve the performance weight of SUs relative to PUs. According to equation (36), the optimal preemption parameter $\left(H^{*}, q^{*}\right)$ can be deduced as follows:

$$
\left(H^{*}, q^{*}\right)=\underset{(H, q)}{\arg \max }\{U(H, q)\} .
$$

Besides, to observe the influence of PU packets' throughput rate and SU packets' throughput rate on system utility from a unified perspective, we deal with these two performance indicators by the max-min normalization method in experiment of Subsection 5.2, and the following is the formula:

$$
x^{*}=\frac{x-x_{\min }}{x_{\max }-x_{\min }} .
$$

Because the preemption probability $q$ is a continuous variable, we cannot precisely calculate the optimum preemption probability $q^{*}$ of the system. By referring to [21], we use SOA to get the optimal preemption probability $q^{*}$. In addition, the preemption threshold is a discrete variable with countable values, so we can firstly optimize the preemption probability to get each maximum system utility $U\left(H, q^{*}\right)$ under all different preemption thresholds. Then we can compare all these obtained system utilities $U\left(H, q^{*}\right)$ and traverse to find the optimal results $\left(H^{*}, q^{*}\right)$ which can realize the total maximum system utility $U\left(H^{*}, q^{*}\right)$. The steps of solving the optimal preemption probability by SOA are shown in Table 1 .

5.2. Optimization Experiment. In order to evaluate our proposed optimization scheme in Subsection 5.1, MATLAB is used to carry out the experiment and get the relevant numerical results. The following are some of the experiment's constant parameters: the amount of channels is $M=6$, SU packets' arrival rate is $p_{2}=0.6, \mathrm{PU}$ and $\mathrm{SU}$ packets' service rates are $\mu_{1}=0.05$ and $\mu_{2}=0.1$, and the two kinds of perception error rates are $f_{1}=0.15$ and $f_{2}=0.1$. Considering the high priority of PU packets, we set $\alpha_{1}$ slightly larger than $\alpha_{2}$, where $\alpha_{1}=10000$ and $\alpha_{2}=9995$.

Figures 12 and 13 show the trends of the system utility corresponding to each preemption threshold with respect to the preemption probability under different PU packets' arrival rates.

From Figures 12 and 13, we can find that when the threshold falls below a certain value, the system utility firstly increases and then decreases with the growth of preemption probability. It can be explained that, in the rising stage, PU packets' throughput rate, which has a greater effect on system utility than SU packets' throughput rate, is the most significant factor impacting system utility. In the descending stage, the opposite is true. However, when the threshold exceeds a certain value and PU packets' arrival rate is large, the system utility hits its optimum when the preemption probability is greatest. This is because a large preemption threshold will greatly affect the preemption behavior of PU packets. However, the main factor affecting the system utility is the performance of PU packets at this stage; therefore a highest preemption probability should be set to alleviate the impact of preemption threshold on PU packets.

As we can see from Table 2, when $p_{1}=0.4$, the maximum system utility is $U\left(H, q^{*}\right)=10002.9824$, and the corresponding optimal preemption parameter value is $\left(H^{*}, q^{*}\right)=$ $(2,0.5014)$; when $p_{1}=0.6$, the maximum system utility is $U\left(H, q^{*}\right)=10000.8266$, and the corresponding optimal preemption parameter value is $\left(H^{*}, q^{*}\right)=(1,0.5345)$.

Table 2 also shows that when the preemption threshold is kept unchanged, the one with a larger PU packets' arrival rate has a greater optimal preemption probability. This is because when other parameters remain unchanged, the 
TABLE 1: Steps to obtain the best preemption probability $q^{*}$ through SOA.

Initialize the number of individuals $n$, change regulator $f_{c}$, spiral shape control factors $u$ and $v$, maximum number of iterations Step 1 iter $_{\text {max }}$, lower bound of the preemption probability variance scope $q_{l}$, and upper bound of the preemption probability variance scope $q_{u}$

Step 2

Set the initial iteration number $x$ to 0 , and set a random preemption probability $q_{i}$ for each individual $i$. The value satisfies $q_{i} \in\left[q_{l}, q_{u}\right]$

Step 3 The system utility of each individual under the corresponding preemption probability $q_{i}$ is calculated by equation (36)

Step 4 Calculate and record the current optimal system utility $U_{m}^{i}$ and the corresponding preemption probability $Q_{m}^{i}$ from $n$ individuals Step 5 Compare and record the global optimal system utility $U_{m}$ and the corresponding preemption probability $Q_{m}$ Update the preemption probability $q_{i}$ of each individual $i$ according to the following formulas:

Step 6

$$
\begin{aligned}
& C_{s}=q_{i} \times f_{c} \times\left(1-x / \text { iter }_{\max }\right), \\
& M_{s}=2 \times f_{c}^{2} \times\left(1-x / \text { iter }_{\max }\right)^{2} \times \operatorname{rand}(0,1) \times\left(Q_{m}^{i}-q_{i}\right), \\
& D_{s}=\left|C_{s}+M_{s}\right|, \\
& k=\operatorname{rand}(0,2 \pi), \\
& r=u \times e^{k \times v}, \\
& q_{i}=r^{3} \times \cos (k) \times \sin (k) \times k \times D_{s}+Q_{m}^{i}
\end{aligned}
$$

where rand $(0,1)$ generates the random number between $[0,1]$ and rand $(0,2 \pi)$ generates the random number between $[0,2 \pi]$ The amount of iterations $x$ is added by 1 ; if $x \leq$ iter $_{\max }$, go back to step 3; otherwise, output the optimum preemption probability $q^{*}=Q_{m}$

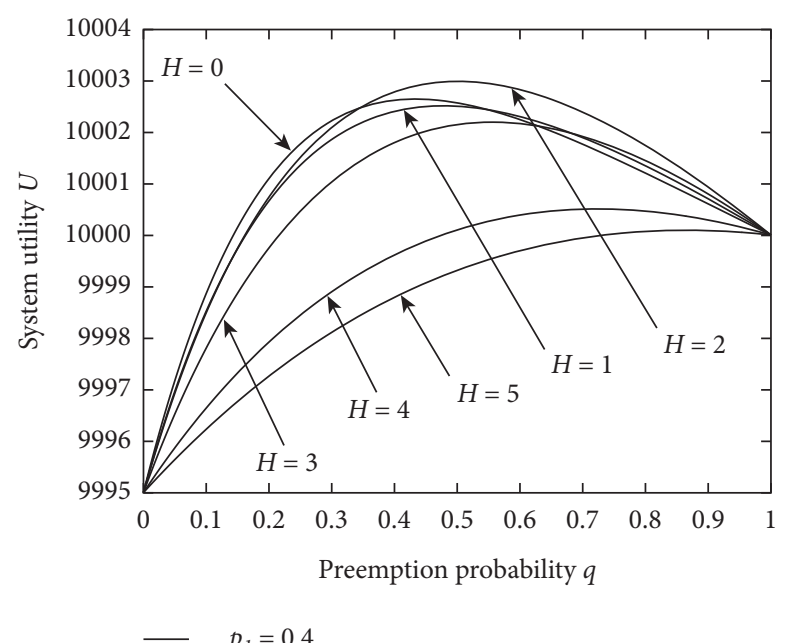

FIGURE 12: The trend of system utility with respect to preemption probability $\left(p_{1}=0.4\right)$.

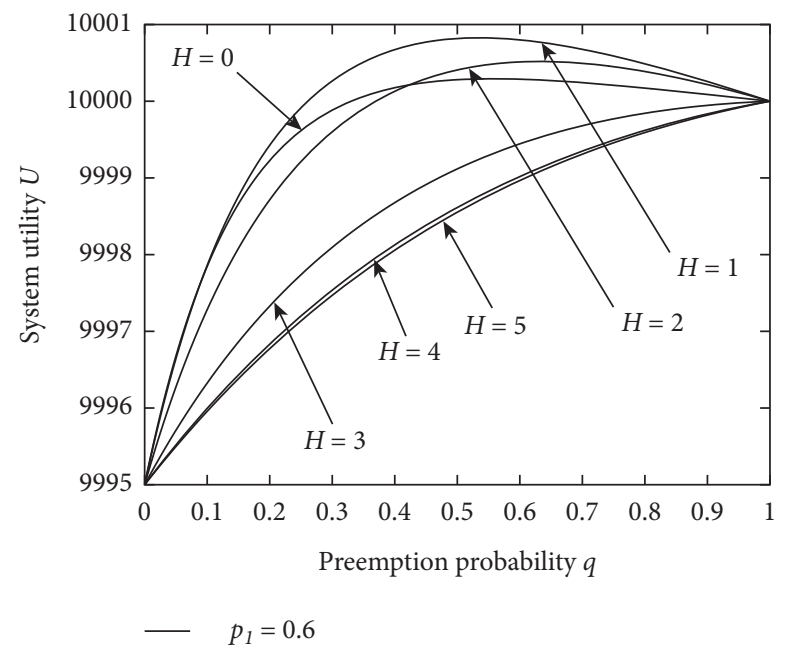

FIGURE 13: The trend of system utility with respect to preemption probability $\left(p_{1}=0.6\right)$. 
TABLE 2: System utility value.

\begin{tabular}{rrrr}
\hline$p_{1}$ & $H$ & $q^{*}$ & $U\left(H, q^{*}\right)$ \\
\hline & 0 & 0.4336 & 10002.6388 \\
& 1 & 0.4778 & 10002.5077 \\
0.4 & 2 & 0.5014 & 10002.9824 \\
& 3 & 0.5556 & 10002.1905 \\
& 4 & 0.7234 & 10000.5058 \\
\hline & 5 & 0.8647 & 10000.0923 \\
\hline & 0 & 0.5570 & 10000.2925 \\
0.6 & 1 & 0.5345 & 10000.8266 \\
& 2 & 0.6317 & 10000.5181 \\
& 3 & 1 & 10000 \\
& 4 & 1 & 10000 \\
& 5 & 1 & 10000 \\
\hline
\end{tabular}

blocking phenomenon of PU packets with a high arrival rate is serious. Therefore, a large preemption probability is needed to alleviate the blocking and improve PU packets' throughput.

\section{Conclusions}

In this paper, two new parameters, preemption threshold and preemption probability, were introduced to centralized CRNs based on overlay sharing mode to control the preemption behavior of PU packets and improve the QoS of SU packets. To have a more detailed overview of the system's performance, considering the case of imperfect spectrum state sensing, a discrete-time queueing model was built in the light of the proposed mechanism for comparison with the traditional multichannel mechanism. Then we got some important performance index expressions about PU and SU packets and made the performance figures under different network environments through numerical experiments. The advantages of our proposed mechanism based on preemption threshold and preemption probability were directly reflected by comparison with the traditional multichannel mechanism. Experimental results illustrated that, under the principle of guaranteeing the QoS of PU packets, the mechanism based on preemption threshold and preemption probability proposed in this paper could significantly reduce the outage rate of SU packets, increase the throughput, and thus improve the SU packets' QoS. Finally, we considered the throughput rate trade-off between PU and SU packets, constructed a performance utility function, and determined the best preemption threshold and the best preemption probability when the system utility is maximum through SOA.

This paper considered a centralized channel management strategy with preemption threshold and preemption probability in cognitive radio networks. As future work, the modeling and performance analysis of cooperative communications with relay nodes in cognitive radio networks will be considered.

\section{Data Availability}

All relevant data are available within the article.

\section{Conflicts of Interest}

The authors declare that there are no conflicts of interest regarding the publication of this manuscript.

\section{Acknowledgments}

This work was supported by the National Natural Science Foundation of China (Grant no. 61701097).

\section{References}

[1] R. Kaur, A. S. Buttar, and J. Anand, "Methods of hybrid cognitive radio network: a survey," in 2018 Second International Conference on Electronics, Communication and Aerospace Technology (ICECA), pp. 1285-1289, Coimbatore, India, March 2018.

[2] J. Liu, S. Jin, and S. Jin, "An imperfect sensing-based channel reservation strategy in CRNS and its performance evaluation," Journal of Industrial and Management Optimization, vol. 16, no. 3, pp. 1149-1169, 2020.

[3] M. El Tanab and W. Hamouda, "Resource allocation for underlay cognitive radio networks: a survey," IEEE Communications Surveys \& Tutorials, vol. 19, no. 2, pp. 1249-1276, 2017.

[4] J. M. Kumbhar and V. P. Kulkarni, "Channel selection in multi-channel multi-user RF energy harvesting cognitive radio networks," in 2018 4th International Conference for Convergence in Technology (I2CT), pp. 1-5, Mangalore, India, October 2018.

[5] S. S. Nair, S. Schellenberg, J. Seitz, and M. Chatterjee, "Hybrid spectrum sharing in dynamic spectrum access networks," in The International Conference on Information Networking 2013 (ICOIN), pp. 324-329, Bangkok, Thailand, January 2013.

[6] S. Mei, B. Chen, F. Hu, and Z. Ma, "Hybrid network coding scheme in cognitive radio networks with multiple secondary users," IEEE Access, vol. 6, pp. 63948-63957, 2018.

[7] N. M. E. Azaly, E. F. Badran, H. N. Kheirallah, and H. H. Farag, "Centralized dynamic channel reservation mechanism via SDN for CR networks spectrum allocation," IEEE Access, vol. 8, pp. 192493-192505, 2020.

[8] H. M. Karaca, "Throughput optimization of multichannel allocation mechanism under interference constraint for hybrid overlay/underlay cognitive radio networks with energy harvesting," Electronics, vol. 9, no. 2, p. 28, 2020. 
[9] A. S. Alfa, H. A. Ghazaleh, and B. T. Maharaj, "A discrete time queueing model of cognitive radio networks with multimodal overlay/underlay switching service levels," in 2018 14th International Wireless Communications \& Mobile Computing Conference (IWCMC), pp. 1030-1035, Limassol, Cyprus, June 2018.

[10] P. K. Tang, Y. H. Chew, W. Yeow, and L. C. Ong, "Performance comparison of three spectrum admission control policies in coordinated dynamic spectrum sharing systems," IEEE Transactions on Vehicular Technology, vol. 58, no. 7, pp. 3674-3683, 2009.

[11] X. Mao, H. Ji, V. C. Leung, and M. Li, "Performance enhancement for unlicensed users in coordinated cognitive radio networks via channel reservation," in 2010 IEEE Global Telecommunications Conference GLOBECOM 2010, pp. 1-5, Miami, FL, USA, December 2010.

[12] N. M. El Azaly, E. F. Badran, H. Nadir Kheirallah, and H. H. Farag, "Performance analysis of centralized dynamic spectrum access via channel reservation mechanism in cognitive radio networks," Alexandria Engineering Journal, vol. 60, no. 1, pp. 1677-1688, 2021.

[13] A. T. El-Toukhy and H. Arslan, "Enhancing the performance of low priority sus using reserved channels in crn," IEEE Wireless Communications Letters, vol. 9, no. 4, pp. 513-517, 2020.

[14] A. U. Khan, G. Abbas, Z. H. Abbas, T. Baker, and M. Waqas, "Spectrum efficiency in crns using hybrid dynamic channel reservation and enhanced dynamic spectrum access," Ad Hoc Networks, vol. 107, p. 16, Article ID 102246, 2020.

[15] T. E. Fahim, A. Y. Zakariya, and S. I. Rabia, "A novel hybrid priority discipline for multi-class secondary users in cognitive radio networks," Simulation Modelling Practice and Theory, vol. 84, pp. 69-82, 2018.

[16] Y. Zhao, W. Yue, and Z. Saffer, "Spectrum allocation strategy with a probabilistic preemption scheme in cognitive radio networks: analysis and optimization," Annals of Operations Research, vol. 47, 2021.

[17] Y. Zhao, H. Li, and J. Liu, "Performance analysis and optimization of CRNS based on fixed feedback probability mechanism with two classes of secondary users," Mathematical Problems in Engineering, vol. 2019, p. 10, Article ID 9385693, 2019.

[18] A. S. Alfa, Queueing Theory for Telecommunications: Discrete Time Modelling of a Single Node System, Springer, New York, NY, USA, 2010.

[19] M. X. Liu and T. F. Xu, "Performance analysis of multichannel mobile communication systems based on discrete time queue," in 2009 Second International Workshop on Computer Science and Engineering, pp. 267-271, Qingdao, China, October 2009.

[20] N. Tian and Z. G. Zhang, Vacation Queueing Models: Theory and Applications, Springer, New York, NY, USA, 2006.

[21] G. Dhiman and V. Kumar, "Seagull optimization algorithm: theory and its applications for large-scale industrial engineering problems," Knowledge-Based Systems, vol. 165, pp. 169-196, 2019. 